\title{
La Société des Etudes Robespierristes, les AHRF et l'espace historiographique italien
}

The Société des études robespierristes, the AHRF and Italian historiography

\section{Anna Maria Rao}

\section{(2) OpenEdition \\ 1 Journals}

Édition électronique

URL : https://journals.openedition.org/ahrf/10867

DOI : 10.4000/ahrf.10867

ISSN : 1952-403X

\section{Éditeur :}

Armand Colin, Société des études robespierristes

\section{Édition imprimée}

Date de publication : 1 septembre 2008

Pagination : 275-293

ISBN : 978-2-200-92515-4

ISSN : 0003-4436

\section{Référence électronique}

Anna Maria Rao, « La Société des Etudes Robespierristes, les AHRF et l'espace historiographique italien », Annales historiques de la Révolution française [En ligne], 353 | juillet-septembre 2008, mis en ligne le 01 septembre 2011, consulté le 01 juillet 2021. URL : http://journals.openedition.org/ahrf/ 10867 ; DOI : https://doi.org/10.4000/ahrf.10867

Ce document a été généré automatiquement le 1 juillet 2021.

Tous droits réservés 


\title{
La Société des Etudes
}

\section{Robespierristes, les AHRF et l'espace historiographique italien}

The Société des études robespierristes, the AHRF and Italian historiography

\author{
Anna Maria Rao
}

1 Le mot « influence » évoquant des courants de réception passive et à sens unique, je préfère parler de rapports d'échanges, et de réseaux de relations scientifiques, institutionnelles et personnelles entre les deux espaces historiographiques français et italien au sujet de la Révolution française ${ }^{1}$.

2 Tout d'abord un constat : 1907, l'année de naissance de la SER en France, est aussi l'année de naissance en Italie de la Société nationale pour l'histoire du Risorgimento, décidée par le Congrès tenu à Milan du 6 au 9 novembre 1906. Elle aussi, comme la SER, publiait une revue, la Rivista storica del Risorgimento ${ }^{2}$. Les différences ne manquaient certes pas : la SER marquait l'opposition entre Aulard et Mathiez autour de Danton et Robespierre ${ }^{3}$; la Société italienne devait servir à unifier les recherches autour de l'histoire du Risorgimento. Mais Révolution française et Risorgimento italien étaient tous les deux considérés comme les moments fondateurs de l'histoire nationale qui passionnait l'historiographie européenne de l'époque.

3 En Italie, le débat et les études sur le Risorgimento renvoyaient forcément à la "grande » Révolution : soit pour souligner l'importance de la décennie 1789-1799 aux origines de l'unification nationale italienne; soit, au contraire, pour souligner les origines autonomes d'un Risorgimento enraciné dans les changements économiques, sociaux, politiques et culturels d'un long XVIII ${ }^{\mathrm{e}}$ siècle, dans lequel la Révolution française aurait provoqué une parenthèse inutile et sanglante ${ }^{4}$. Cette version nationaliste devint dominante pendant le fascisme, quand l'Institut pour l'histoire du Risorgimento qui, en 1935, prit la place de la Société, fut présidé par Cesare Maria de Vecchi di Val Cismon, ministre aussi de l'Éducation nationale. Considérant l'index de la Rassegna storica del Risorgimento des années $1914-1963{ }^{5}$ on constate que, sur à peu près 1870 titres, moins d'une centaine portent sur l'Italie révolutionnaire et napoléonienne, dont une 
quarantaine après 1946, signe de la nouvelle saison d'études qui s'ouvrit après le fascisme et la guerre. Même en ce qui concerne les comptes rendus d'ouvrages sur la Révolution française, il faut attendre les traductions italiennes des années cinquante pour trouver les noms de Jean Jaurès, Georges Lefebvre, Albert Mathiez, Jacques Godechot ${ }^{6}$.

4 Le climat nationaliste n'empêcha pas la parution de plusieurs ouvrages sur la Révolution française d'auteurs italiens ou de traductions d'ouvrages français. Elle n'empêcha pas non plus les rapports entre les AHRF et les revues italiennes.

Dès le début l'Italie est présente dans la Revue de la presse des Annales révolutionnaires, en particulier avec la Nuova antologia et la Nuova rivista storica, mais les comptes rendus d'ouvrages italiens ou concernant l'Italie sont assez rares. Il faut remarquer l'importance accordée, dès sa première édition de 1905, à la Révolution française de Gaetano Salvemini, le premier grand ouvrage de l'historiographie italienne sur la Révolution. En 1909, Albert Mathiez le signalait de façon enthousiaste ${ }^{7}$ :

«Voici un excellent manuel, le meilleur sans contredit qui existe sur l'histoire de la Révolution. Les causes de la chute de l'ancien régime sont exposées dans une forte introduction avec une lucidité, une vigueur et une impartialité remarquables. M. Salvemini ne s'est pas seulement inspiré des travaux les plus autorisés, il en a repensé les conclusions et son œuvre a une valeur originale. Le récit des événements, qu'il termine dans ce premier volume à la proclamation de la république, est clair, serré, vivant. Aucun fait important qui ne soit mis en lumière et expliqué dans ses causes et dans ses conséquences, aucun acteur du drame qui ne soit présenté au bon moment. C'est merveille de suivre un guide aussi intelligent et un conteur aussi agréable ».

6 Il exprimait toute son admiration pour une méthode et une écriture historiques capables d'harmoniser l'érudition avec la vigueur de l'interprétation, la clarté de l'exposition avec le goût de la narration. Salvemini, observait-il, était « de l'école de Tocqueville » : «Il se dit que les faits historiques ne valent pas la peine d'être rapportés si on ne s'attache pas à en découvrir l'explication et l'enchaînement. Il philosophe donc à tout instant, mais sans le moindre pédantisme, et sans perdre jamais le contact des réalités $»^{8}$. Il ne manquait pas de soumettre à l'auteur quelques points de désaccord et les nombreuses fautes d'impression. Il lui reprochait d'avoir trop grandi le rôle de Mirabeau, d'avoir négligé la politique religieuse des Constituants et, surtout, d'avoir fait « un grand usage des travaux et des publications de M. Aulard» sans «une suffisante critique » :

«C'est ainsi qu'il épouse les aversions anti-robespierristes de l'école ; qu'il prétend que l'agitation contre le marc d'argent fut une agitation superficielle, qu'il voit dans toutes les crises la main invisible de Danton, qu'il croit que le seul Marat protesta contre la loi Chapelier, qu'il croit que l'académicien Condorcet jouissait auprès de la masse des révolutionnaires d'une réelle autorité ${ }^{9}$, qu'il assure que les soldats de la Révolution n'avaient pas le temps ni le goût de lire les journaux ».

7 Trop près d'Aulard et trop loin de Mathiez, donc, le livre de Salvemini. Malgré ceci, ce compte rendu le consacrait comme un ouvrage fondamental. Mathiez reprenait son jugement et ses réserves en 1926, à la parution de la cinquième édition de «cet excellent manuel ", "à l'heure même", ajoutait-il, "où l'auteur est obligé de se réfugier en France pour échapper à la tyrannie »: «Bien que les préventions de M. Salvemini contre Robespierre se soient atténuées, bien que son admiration ancienne pour Danton ait à peu près disparu, je ne puis pas encore souscrire à tous ses 
jugements ». Ses critères d'évaluation dépendaient donc du « degré » de robespierrisme et du rôle assigné aux luttes entre les partis ${ }^{10}$.

Salvemini mis à part, on ne trouve dans les Annales révolutionnaires et dans les AHRF que quelques notices éparses sur quelques ouvrages italiens. Jusqu'à la mort de Mathiez, la revue italienne la plus régulièrement citée est la Nuova rivista storica, fondée en 1917 par Corrado Barbagallo, qui la dirigea jusqu'en $1930^{11}$.

Dans le numéro des AHRF de 1931 écrit en hommage à Albert Mathiez, Barbagallo faisait remonter à 1911 sa rencontre avec l'historien français ${ }^{12}$ :

"L'occasion qui m'a donné la fortune de rencontrer Albert Mathiez, fut un article d'un des collaborateurs de la Nuova rivista storica de 1911, que je dirigeais alors long article d'un savant qui, lui aussi, est aujourd'hui disparu. Dans cet article il soutenait une thèse diamétralement opposée à celle que Mathiez avait commencé d'exposer dans ses livres célèbres sur la Révolution ".

L'auteur de cet article, dont il ne disait pas le nom, était Carlo Alberto Alemagna, un enseignant de Salerne, qui écrivait sous le pseudonyme de général Filareti : un amateur plus qu'un savant, mais que Barbagallo avait beaucoup apprécié. Son essai, Danton et Robespierre, paru dans la revue en 1910-1911 et réimprimé en 1911 avec une introduction du même Barbagallo, appliquait à l'histoire les principes de la « psychologie sociale». Prenant parti pour Aulard contre Mathiez, il faisait de Danton son héros et traçait un portrait de Robespierre fondé sur les caractères les plus venimeux de la propagande contre-révolutionnaire et de l'anthropologie criminelle: frigide, méfiant, autoritaire, fanatique, démagogue cérébral, rationaliste abstrait. Un portrait qu'il rapprochait de celui de Lénine, étant tous les deux des «liquidateurs » ${ }^{13}$.

11 On peut s'étonner qu'un tel essai inaugurât les rapports intellectuels entre Mathiez et Barbagallo. Celui-ci affirmait aussi que l'article de Filareti avait fait connaître Mathiez en Italie :

"C'était la première fois, je crois, que ses interprétations originales étaient soumises aux savants italiens, mais c'était peut-être aussi une des rares occasions où elles furent amplement diffusées, et très vivement réfutées avec une chaleur égale à celle qui était mise par le savant français à les affirmer. De cette occasion unique, naquit notre amitié intellectuelle, et, dès lors, tout nouveau livre, tout article de la Nuova Rivista Storica, touchant à la Révolution, devint l'objet d'un dialogue critique dans lequel il en vint peu à peu à me conquérir, non seulement à une sincère admiration sur son œuvre, mais aussi à une partie de ses convictions ».

12 À l'époque de son hommage à Mathiez, Barbagallo n'était plus le directeur de la Nuova rivista storica, tout en continuant à y collaborer, et Filareti était mort depuis 1917. Mais Barbagallo partageait encore son jugement négatif sur Robespierre. Les opinions de Mathiez, écrivait-il, «étaient faites de la matière même inattaquable et incorruptible dont sont constituées les grandes fois : matière qu'il n'est possible ni de fléchir, ni de détruire». Son but principal avait été «la réhabilitation de Robespierre», ce qui impliquait « le renversement de Danton ». Il avait élargi le panorama à tous les partis et les hommes de la Révolution, les girondins, les indulgents, les jacobins, les montagnards. Mais s'il avait réussi «à abaisser Danton » il n'avait pas pu «élever d'un pouce » la figure de Robespierre, tout en confirmant son incorruptibilité :

«Robespierre ne fut pas un homme, ce fut une idée sans entrailles, qui, pour le triomphe d'une abstraite algèbre politique, poursuivie cependant avec le désintéressement le plus complet, jeta sans pitié la France dans une mer de sang et de haines. Avoir voulu réaliser à l'aide de ces systèmes, une parfaite, libre démocratie politique et sociale, voilà son erreur capitale et celle du parti qui l'a 
suivi. Ce jugement n'est pas le nôtre, c'est la conclusion qui se dégage des pages des deux derniers livres de Mathiez: La Révolution française et La vie chère et le mouvement social sous la Terreur $»^{14}$. l'intérêt pour l'histoire économique et sociale ; le lien entre la Révolution française et le Risorgimento italien, qu'il continua à défendre au moment même où les institutions culturelles fascistes le rejetaient. La Nuova rivista storica signala constamment ce qui se publiait en France sur la période révolutionnaire. En 1927, l'année même de sa parution, elle donnait une notice sur La vie chère et le mouvement social sous la Terreur de Mathiez; l'année suivante, Barbagallo citait ce dernier en termes élogieux dans sa postface à un autre article du général Filareti ${ }^{19}$. Mieux connu en France que Croce ${ }^{20}$, Barbagallo trouva chez Mathiez et dans les AHRF la même attention à ses œuvres et à sa revue, la seule revue italienne presque toujours présente dans la Revue de la presse jusqu'en 1931, tandis que les autres - Nuova antologia, Giornale storicodellaletteraturaitaliana, RassegnastoricadelRisorgimento, Rivistastoricaitaliana -étaient citées de façon plus épisodique.

17 À signaler, en particulier, la présentation flatteuse que Mathiez donnait en 1919, l'année même de sa parution, du livre de Barbagallo sur les origines de la grande industrie ${ }^{21}$ :

«Voici un manuel excellent, clair, méthodique, d'inspiration réaliste, sans théories préconçues, informé enfin des dernières études. M. Barbagallo, dont l'intelligence est perpétuellement en éveil et la curiosité universelle, n'est pas dupe des formules toutes faites qui prétendent enfermer la naissance de la grande industrie dans le machinisme d'une part et la liberté commerciale de l'autre. Il a bien vu que l'époque des grandes guerres de la Révolution et de l'Empire a contribué puissamment à donner une physionomie particulière à l'évolution industrielle des deux grandes 
antagonistes, la France et l'Angleterre, qui sont au centre de son exposé. Il sait que les systèmes d'idées ne dominent pas l'histoire, mais qu'ils ne sont qu'une représentation souvent tardive des intérêts concrets ${ }^{22}$ française de Carlo Morandi, lui aussi parmi les collaborateurs de la Nuova rivistastorica ${ }^{23}$. En 1931 Mathiez dédiait une notice au « petit livre clair et alerte » de Mario Di Gennaro sur John Law, préfacé par Barbagallo ${ }^{24}$. La même année, un bref commentaire soulignait l'utilité de l'article de Baldo Peroni sur l'occupation française de la Lombardie en 1796-1797, tout en critiquant son attitude nationaliste :

«L'exposé semble empreint d'une hostilité systématique contre l'occupation française. L'auteur reproche à Bonaparte d'avoir agi en chef et en vainqueur, comme s'il pouvait en être autrement en temps de guerre et comme si les patriotes italiens eussent été en état, par leurs seules forces, de libérer le pays de la domination autrichienne! Il exagère l'importance du mouvement national. Il reconnaît cependant que les idées républicaines ne purent pas se répandre largement dans les masses populaires parce qu'elles se présentaient toujours associées à des violences contre l'Église. Son étude nourrie de documents inédits, sera certainement très utile $»^{25}$.

Peroni, qui collaborait régulièrement à la Nuova rivista storica, ne méritait pas ces reproches, puisqu'il soutint toujours l'importance de la Révolution française pour le Risorgimento italien. En 1936 il fournira un instrument de recherche précieux par son inventaire des sources pour l'histoire d'Italie entre 1789 et 1815 existant dans les Archives parisiennes ${ }^{26}$.

Il est impressionnant de voir comment Mathiez était attentif même aux plus petites publications ou aux ouvrages d'auteurs peu connus (aujourd'hui injustement ignorés) pourvu qu'ils offrissent aux chercheurs des documents intéressants ${ }^{27}$. On peut citer encore le cas de Raffaele Ciampini, un nom presque disparu de l'histoire de l'historiographie italienne. Professeur à l'Université de Florence, Ciampini (né en 1895) sera à la fin des années 1950 parmi les fondateurs du Centre national d'études napoléoniennes de Portoferraio, dans l'île d'Elbe. Nous lui devons la présentation du premier numéro du Bollettino italiano di studi napoleonici de $1962{ }^{28}$. Ses études napoléoniennes ne furent pas beaucoup appréciées par Mathiez. En 1930, dès sa parution, il signalait son livre Napoleone visto dai contemporanei ${ }^{29}$, "une sorte de biographie épique de Napoléon répartie en cinq chapitres dont chacun est basé sur un mémorialiste différent ». Thibaudeau y était " porté aux nues », tandis que Bourrienne était considéré comme un plagiaire et un dénigreur systématique dont il faut se méfier, ce qui ne l'empêche pas de l'utiliser abondamment». Le jugement final n'était pas flatteur :

«Mais comment M.C. choisit-il ce qu'il garde et ce qu'il rejette ? Son critérium est simple. Quand le mémorialiste dit du bien de Napoléon, il dit la vérité, quand il le critique ou le présente en fâcheuse posture, il devient un menteur ou tout au moins il est suspect de malveillance. ${ }^{30}$ »

Jusqu'à la mort de Mathiez, donc, les rapports entre les AHRF et l'historiographie italienne sont liés surtout à la Nuova rivista storica et à Corrado Barbagallo. Après 1931 ces échanges paraissent plus rares et difficiles. Depuis 1930 Barbagallo n'a plus la direction de la revue, confiée à Gino Luzzatto, dont les intérêts sont plus éloignés de la Révolution française. Promoteur convaincu de l'histoire économique et sociale, Luzzatto continue à faire de la revue qu'il dirige un espace de liberté, jusqu'en 1938, quand il est frappé par les lois raciales ${ }^{31}$. 

En 1939 il rend compte du livre de Giuseppe Maranini, Classe e stato nella rivoluzione francese, paru en $1935^{32}$. L'année 1940, la dernière des AHRF avant la longue suspension imposée par la guerre, voit une présence remarquable de l'historiographie italienne. Le livre de Raffaele Ciampini sur la chute de la monarchie, paru en $1934^{33}$, est jugé bien mieux que le précédent, pour son «excellente information " et "par les documents inédits qu'il utilise $»^{34}$. Lefebvre signale aussi l'édition par le même Ciampini des lettres du toscan Filippo Mazzei de 1788-1791, à l'époque où il était l'agent du roi de Pologne à Paris $^{35}$ et une étude de Ugo Lenzi ${ }^{36}$. Les éloges ne manquent pas envers l' «importante étude » de Pia Onnis Rosa sur Filippo Buonarroti publiée dans la Rivista storica italiana de 1937 : «On rapprochera ce travail du chapitre consacré à l'activité de Buonarroti dans la thèse de M. J. Godechot que M. Onnis n'a pu connaître " $^{37}$.

Pia Onnis Rosa fut à son tour un intermédiaire entre les deux historiographies, d'autant plus qu'elle alla à Paris pour ses recherches sur Buonarroti. Il n'est pas étonnant de la retrouver dans les AHRF à leur reprise après la guerre, avec son article sur les études italiennes sur l'histoire de la Révolution française de 1940 à 1949 ${ }^{38}$. Dans cet article elle reliait directement l'intérêt scientifique pour l'histoire de la Révolution à la passion politique de la liberté. Elle rappelait le volume de l'Histoire universelle de Corrado Barbagallo dédié à la révolution américaine et à la révolution française, les études d'Armando Saitta et Felice Battaglia sur les constitutions et les déclarations des droits françaises, le livre de Franco Venturi sur l'historiographie de la Révolution, le volume de Cantimori sur Utopisti e riformatori italiani de 1943, les études sur Babeuf et Buonarroti et les textes édités par Gastone Manacorda, Giuseppe Berti, Armando Saitta, Alessandro Galante Garrone, La chute de la monarchie de Raffaele Ciampini. Elle soulignait ainsi les conditionnements politiques exercés sur les interprétations historiographiques :

«En effet le nationalisme fasciste voulait nier les connexions entre la Révolution française et le Risorgimento, en exaltant les insurrections populaires et antifrançaises comme une expression du patriotisme, tandis que les conspirations italiennes en faveur de la pénétration révolutionnaire figuraient comme des actes de trahison en connivence avec l'étranger ${ }^{39}$.

La reprise des $A H R F$ coïncide donc avec une importante reprise des études italiennes sur la période révolutionnaire qui se reflète immédiatement dans la richesse des notices et des comptes rendus, parmi lesquels, à côté de Lefebvre, on retrouve de plus en plus Jacques Godechot. En 1949 celui-ci commente l'étude de Pia Onnis sur Filippo Buonarroti commissaire à Oneglia, publiée dans la Nuova rivista storica de 1939, expliquant que " les événements » lui avaient « empêché de rendre compte plus tôt de cet intéressant article », qui complétait celui de $1937^{40}$.

Si Pia Onnis Rosa recueillait en quelque sorte l'héritage des rapports entre Mathiez et Barbagallo, d'autres représentaient une historiographie nouvelle, nourrie par l'exil et par l'antifascisme.

26

«Franco Venturi a écrit sur les origines de l'Encyclopédie un excellent petit livre ${ }^{41}$ : Lefebvre signalait ainsi en 1949 les premiers fruits de cette historiographie. Pendant son exil en France, Venturi s'était adonné à l'étude non seulement de l'Encyclopédie mais aussi de l'historiographie révolutionnaire. Dans le même numéro de 1949 Lefebvre rendait compte de son livre sur les historiens de la Révolution ${ }^{42}$, « essai panoramique sur le développement des études révolutionnaires depuis un demi-siècle », dont plus de 
la moitié consacré à Jaurès, aux rapports entre ses idées politiques et son interprétation de la Révolution ${ }^{43}$.

À côté de Venturi, Alessandro Galante Garrone : Venturi lui-même le met en relation avec Lefebvre, et celui-ci le pousse à se pencher sur Gilbert Romme ${ }^{44}$. Sa première lettre à Galante Garrone est du 10 août $1947^{45}$. En 1950 Lefebvre publie un long compte rendu de son Buonarroti e Babeuf, paru en 1948. Galante Garrone avait « bien mis en lumière les caractéristiques respectives des deux personnages ». Grâce à "l'étude minutieuse des documents » il pouvait écarter le doute que l'Histoire de la conjuration de Buonarroti, publiée en 1818, ne soit pas l'exposé fidèle du programme élaboré trente ans auparavant; il abordait le problème de leur communisme et de son rapport avec la société rurale qu'ils avaient connue, corse pour l'un, picarde pour l'autre. Lefebvre suggérait de développer la recherche sur la notion de dictature révolutionnaire que l'auteur avait laissée de côtét ${ }^{46}$.

L'année 1951 vit paraître dans les AHRF deux comptes rendus du livre de Saitta sur Buonarroti, l'un de Jacques Godechot, l'autre de Georges Lefebvre ${ }^{47}$. Godechot concluait son analyse par une considération générale sur l'historiographie italienne du moment : "Par cet aperçu, il est possible de juger de la richesse de ce livre qui fait honneur à son auteur et à la jeune école historique italienne $»^{48}$. Voici en 1954 encore un compte rendu de Lefebvre à la traduction italienne de Quinet par Galante Garrone chez Einaudi en 1953. Il appréciait beaucoup l'introduction de son collègue italien, mais il observait que Quinet ne gardait d'intérêt que " comme témoignage de l'état d'esprit qui régnait en son temps, dans une partie des classes dominantes $»^{49}$.

Les liens entre les deux historiographies se traduisirent enfin par une présence plus significative des historiens italiens dans la SER. Dans son bilan de 1958 sur cinquante ans d'histoire de la Société, Maurice Dommanget ne donnait pas de précisions sur les membres étrangers et sur les abonnés à la revue à ses débuts : "La Société rayonnait déjà sur 9 pays et comptait des adhérents dans les deux mondes $»^{50}$. Il signalait 7 adhérents et abonnés en Italie en 1925, 16 en $1957{ }^{51}$ et soulignait l'ouverture de la revue à la collaboration des étrangers : pour l'Italie, Gian Battista Schifalacqua et Pia Onnis $^{52}$. En avril 1952 Alessandro Galante Garrone fut élu membre du Comité ${ }^{53}$. Il y resta jusqu'à sa mort. En même temps, Armando Saitta devint un des interlocuteurs les plus importants de la Société et de la revue, grâce à ses rapports de collaboration et d'amitié avec Lefebvre et Godechot, et bientôt Suratteau et Soboul ${ }^{54}$. Membre du Comité directeur de la Société des études robespierristes en $1960^{55}$, Saitta en fut le viceprésident en 1971 à la place d'Alfred Rufer. Membre de la Commission internationale d'histoire de la Révolution française dès sa création, en 1976, en 1985 il en sera le viceprésident ${ }^{56}$.

30 Lefebvre continua à suivre avec attention l'historiographie italienne jusqu'à sa mort. Dans son hommage de 1960, Galante Garrone donnait toute une liste de comptes rendus et de notices, «souvent accompagnées d'observations critiques » rédigées par Lefebvre, qui « lisait couramment l'italien $»^{57}$. Parmi ces notices, il signalait l'examen de l'article d'Armando Saitta, «Il robespierrismo di Filippo Buonarroti e le premesse dell'unità italiana ", extrait de la revue Belfagor de 1955, publié dans les AHRF de $1957^{58}$. C'est une notice importante, sous plusieurs points de vue. Avant tout, elle confirme l'importance des liens personnels et des échanges scientifiques: «L'Italie se voit remarquablement représentée parmi les tirages à part qui nous ont été envoyés » ${ }^{59}$. Deuxièmement, elle se faisait écho des débats en cours dans l'historiographie italienne entre Franco Venturi et 
Armando Saitta autour du jacobinisme italien. Finalement il connectait, encore une fois, les recherches italiennes et les recherches françaises, écrivant, à propos de la thèse de Saitta «qu'on ne peut séparer de la Révolution française les débuts du mouvement unitaire » : « C'est une thèse qui, je présume, ne peut déplaire à Jacques Godechot, qui apporte de nouvelles contributions à l'histoire de l'Italie directoriale et impériale $»^{60}$. Le même numéro signalait l'étude de Carlo Zaghi sur le Directoire et l'Italie de 1954 et l'étude de Pasquale Villani sur Zurlo de $1955^{61}$.

31 Désormais, la production italienne était signalée et commentée surtout par Godechot: le nouveau livre de Galante Garrone sur Buonarroti ${ }^{62}$, le deuxième volume de celui de Saitta ${ }^{63}$, les Giacobini italiani de Delio Cantimori ${ }^{64}$, le Gilbert Romme de Galante Garrone ${ }^{65}$, les études d'histoire économique et sociale de Bologne d'Umberto Marcelli ${ }^{66}$.

C'est encore Godechot qui rédige la nécrologie de 1953 de Benedetto Croce, que les $A H R F$ avaient presque ignoré jusqu'alors. Il se rappelait de l'avoir rencontré à Naples en 1933 : «Alors persécuté par le fascisme [...] il me dit son amour pour la France et son espoir dans le triomphe de la démocratie ». Il résumait ainsi sa conception historique, qu'il ne partageait, évidemment, pas: "Croce, qu'on a surnommé le "pape du libéralisme", ne croyait pas au matérialisme historique, il accordait, au contraire, une influence capitale à l'action des idées ». Il avait «grandement contribué » au "grand essor pris en Italie, surtout depuis 1944, par les recherches historiques ${ }^{67}$.

Les historiens français de la Révolution étaient frappés par l'attention que les études italiennes portaient aux questions historiographiques. À ce sujet leur dialogue avec leurs collègues italiens ne manquait pas d'incompréhensions.

Galante Garrone l'écrivait de façon efficace dans son hommage à Lefebvre de 1960. Après avoir évoqué les raisons politiques de l'intérêt des historiens italiens de son époque pour la Révolution française ${ }^{68}$, qui l'avaient poussé à traduire l'ouvrage de Quinet, il rappelait que Georges Lefebvre lui avait manifesté « sa surprise qu'on publiât en Italie un ouvrage désormais si pauvre d'intérêt pour l'“histoire positive" »:

«Il ne pouvait se rendre compte que, chez nous, le fascisme avait créé la nécessité de recommencer derechef, de remonter aux sources libérales et démocratiques du XIXe siècle, de comprendre - dans sa valeur éternelle et dans ses limites contingentes -, ce que Quinet et Croce avaient appelé la "religion de la liberté". Il trouvait aussi un peu bizarre que chez nous, sous l'influence de Croce, se manifestât un goût si vif et si persistant pour l'histoire de l'historiographie » ${ }^{69}$.

Dans la période entre les deux guerres, les références communes au matérialisme historique et plus généralement à l'histoire économique et sociale avaient permis à Mathiez et à Barbagallo de se rencontrer et de dialoguer, malgré toutes leurs divergences sur Robespierre. Après la guerre, les historiens français de la Révolution ne semblaient apercevoir dans l'historiographie italienne que du Croce et de l'histoire des idées. Lefebvre considéra avec surprise mais avec intérêt les ouvrages d'histoire politique et d'histoire de l'historiographie de Franco Venturi, de Galante Garrone, d'Armando Saitta. Albert Soboul garda la même attitude ${ }^{70}$.

Jacques Godechot, au contraire, non seulement ne comprit pas toujours les raisons de l'histoire politique, mais il ne reconnut pas immédiatement les efforts de renouvellement qu'une nouvelle génération d'historiens fera entre les années 1950-1960 à l'exemple de la France, pratiquant justement des recherches d'histoire économique et sociale, très souvent en contradiction explicite avec la tradition de B. Croce. 
37 Lisons, par exemple, ses considérations sur " Le congrès franco-italien de l'île d'Elbe ", organisé à Portoferraio en mai 1954 par la Société toscane pour l'histoire du Risorgimento et dédié aux rapports entre la France et l'Italie à l'époque révolutionnaire et impériale. Il y participait avec une dizaine d'autres collègues français et une centaine d'italiens, «parmi lesquels nos amis Galante Garrone, Pieri et Vaccarino de Turin, le professeur Ghisalberti de Rome, etc. [...]». Godechot signalait «l'intéressante communication de M. Galante Garrone sur la traduction des codes français en italien par Giovanni Gambini, d'après les papiers de ce traducteur retrouvés fortuitement à Bâle par M. Garrone ", mais, de façon générale, il observait :

«Il nous est apparu que l'historiographie italienne était encore très orientée vers l'histoire politique et l'histoire intellectuelle, et ne s'attachait pas assez à l'examen des structures politiques et sociales. Nous avons essayé de montrer que le régime révolutionnaire et impérial avait entrainé la formation en Italie d'une classe nouvelle, une petite bourgeoisie de fonctionnaires et d'officiers de l'armée, qui s'est enrichie par l'achat des biens nationaux, le partage des communaux et aussi l'acquisition d'autres terres que l'abolition des fideicommis a remis dans le circuit commercial. Le professeur Nino Cortese, de Naples, a contesté cette interprétation, tandis que le professeur Dal Pane, de Bologne, a pensé - et cela nous semble pertinent - que cette classe nouvelle avait commencé à se former dans certaines régions, par exemple en Toscane, avant même la Révolution. De toutes manières, il serait désirable que des études sérieusement documentées permettent de formuler des conclusions précises [...]».

38 En tout cas, il appréciait beaucoup l'occasion de rencontre qui avait été créée : «C'est au cours de réunions de ce genre, dont on souhaite qu'elles se multiplient, que pourra s'élaborer une collaboration internationale efficace, sans laquelle aucune grande entreprise historique ne saurait réussir ${ }^{71}$.

Une autre occasion importante de rencontre fut pour lui le $\mathrm{X}^{\mathrm{e}}$ Congrès international des sciences historiques tenu à Rome en septembre 1955. Godechot observait l'importance qui y avait assumé l'opposition entre les tenants de l'histoire événementielle et « les partisans d'une histoire plus structurale qui se groupent autour des "Annales" de Lucien Febvre ${ }^{72}$. Mais il gardera ses réserves et sa méfiance à l'égard d'une historiographie italienne qu'il considérait, à tort, presque totalement inspirée par ce B. Croce qu'il estimait mais dont il n'aimait pas les positions historiographiques.

40 Ainsi, son long compte rendu du livre de Carlo Zaghi sur Bonaparte et le Directoire, de 1956, en reconnaissait tout de suite l'importance : «Voici sans doute le plus important ouvrage relatif à l'histoire diplomatique du Directoire depuis la thèse de Raymond Guyot ». Mais seulement pour exprimer toutes ses perplexités à l'égard de l'histoire diplomatique :

«L'étude de M. Zaghi appartient entièrement à un genre quelque peu délaissé en France depuis une vingtaine d'années : l'histoire diplomatique. Dans ce genre, il est incontestablement une réussite et il complète remarquablement la thèse, pourtant si bien informée, de Raymond Guyot. Il est pourtant permis de se demander si les négociations diplomatiques ont eu sur la marche des événements toute l'influence qu'on leur attribue. La diffusion des idées, la modification des structures sociales, l'évolution de la conjoncture économique n'ont-elles pas dicté aux gouvernements et aux diplomates, la plupart du temps sans qu'ils s'en rendent compte, toutes leurs attitudes? Dès lors est-il logique de consacrer tant d'efforts à une histoire qui ne traduit que l'aspect superficiel des choses, mais qu'on peut atteindre facilement, car de toutes les actions humaines, ce sont les démarches des diplomates qui ont aligné dans les archives les dossiers incontestablement les plus complets et les plus volumineux? Ces réflexions n'enlèvent rien d'ailleurs au mérite de $\mathrm{M}$. Zaghi, et 
quelle que soit l'opinion que l'on professe sur la valeur de l'histoire diplomatique, son livre sera désormais indispensable à quiconque voudra étudier les relations internationales à l'époque Directoire [sic] ${ }^{73}$.

économique et sociale d'après la guerre, l'historiographie italienne paraissait toujours dominée dans les AHRF par l'histoire politique, ce qui avait fait son originalité aux yeux de Lefebvre, sa limite aux yeux de Godechot. Cet aspect était destiné à redevenir ensuite une raison d'éloge et d'attraction, lorsque les AHRF elles-mêmes reviendront, pour ainsi dire, à une histoire politique qu'elles n'avaient, en vérité, pas du tout négligée. Il suffit de lire l'hommage de Markov et Suratteau à Saitta après sa mort, en $1991^{77}$.

"Armando Saitta a gardé jusqu'au bout une originalité [...] caractéristique de la
formation des historiens italiens et, à moindre degré de celle des historiens
allemands, d'être resté un "philosophe de l'histoire", se moquant des modes qui
ont, en France et dans d'autres pays, privilégié successivement l'histoire
économique, puis l'histoire économico-sociale, puis la socio-économique, puis la
démographie historique, puis l'histoire du "mental", etc. Saitta a continué à donner
la primauté à l'histoire des idées - si délaissée en France - et, aussi, à l'histoire
politique. Il n'a pas attendu le revirement que les historiens de la "troisième école
des Annales" ont amorcé ".

Ils soulignaient aussi le fait que, en privilégiant l'histoire politique, Saitta «s'était défait de la pesanteur d'une histoire se disant marxiste ", un choix qui n'avait certes pas lésé l'amitié et le soutien dont il jouissait de la part d'Ernest Labrousse et Albert Soboul : «Ce retour au politique, associé à l'éloge de l'histoire des idées et à la louange de l'historiographie - qui fut la dernière grande passion de notre ami disparu - nous réjouit et justifie, s'il était besoin, l'œuvre de Saitta " ${ }^{78}$. 
L'historiographie italienne et l'historiographie française de la Révolution, partiellement alliées à l'époque de Mathiez par la revendication de l'histoire économique et sociale, semblaient ainsi se rencontrer sur le terrain de l'histoire politique.

\section{NOTES}

1. J'ai déjà examiné quelques aspects de ces rapports, sur lesquels, au moins en partie, je serai obligée de revenir ici : Anna Maria Rao, « Un héritage hors de France : les institutions culturelles italiennes et la Révolution française ", dans Christine Peyrard, Michel Vovelle (dir.), Héritages de la Révolution française à la lumière de Jaurès, Aix-en-Provence, Publications de l'Université de Provence, 2002, p. 131-145; Id., "France et Italie: le jacobinisme en question", dans Martine Lapied, Christine Peyrard (dir.), La Révolution française au carrefour des recherches, préface de Michel Vovelle, Aix-en-Provence, PUP, 2003, p.155-179; Id., «Lumières et révolution dans l'historiographie italienne", AHRF, $\mathrm{n}^{\circ}$ 334, octobre-décembre 2003, p. 83-104; "Alessandro Galante Garrone, historien de la Révolution française », AHRF, $\mathrm{n}^{\circ}$ 344, avril-juin 2006, p . 219-237.

2. Son titre change : Rivista storica del Risorgimento (qui existait déjà), Il Risorgimento italiano, puis Rassegna storica del Risorgimento. Cf. Massimo Baioni, La «religione della patria ». Musei e istituti del culto risorgimentale (1884-1918), Treviso, Pagus, 1994, p. 84-90.

3. Maurice Dommanget, «La Société et les Annales. Cinquante ans d'histoire (1908-1958) », AHRF, 30, 1958, p. 6-27.

4. Sur ces arguments et les différentes nuances de l'historiographie nationaliste je renvoye aux textes cités dans la note 1.

5. Giovanna Bernau, Ada Guatelli (dir.), Rassegna storica del Risorgimento, Indice per autori e per materie 1914-1963, Roma, Istituto per la storia del Risorgimento italiano, 1968.

6. Seul Godechot avait déjà paru pour l'ouvrage écrit avec Georges Bourgin, L'Italie et Napoléon, dont le compte rendu (de Marino Ciravegna) fut publié dans l'année XXIII, 1936, p. 17171719. La revue publia aussi Jacques Godechot, "Démographie et économie dans les origines du Risorgimento », dans l'année XLIY 1957, p. 382-389.

7. Annales révolutionnaires, II, 1909, p. 607-609.

8. Il poursuivait : « Amoureux des idées, il l'est aussi des précisions. Les statistiques et les chiffres viennent toujours à l'appui de ses raisonnements, et ses raisonnements n'interrompent jamais le fil du récit. Je n'en finirais pas si je voulais signaler toutes les pages pénétrantes qui m'ont arrêté dans ce livre $[. .]$.$» .$

9. À ce sujet il le renvoyait à son article de 1905 dans la Revue critique.

10. AHRF, n.s., III, 1926, p. 198 : « Le parti populaire et démocratique dont Robespierre fut le chef incontesté n'apparait pas assez dans cet ouvrage, pas plus d'ailleurs que le parti opposé, le parti feuillant qui obéissait à la double direction des Fayettistes et des Laméthistes. Les luttes des partis ou plutôt des clientèles qui les composaient sont restées à l'arrière plan. M. Salvemini a sans doute amélioré son exposé des questions religieuses et des questions financières. Mais la vie chère et ses répercussions de toute sorte auraient mérité un peu plus d'attention Il est vrai qu'on ne peut pas traiter à fond en 350 pages tous les problèmes d'un sujet aussi vaste. Constatons avec reconnaissance que cette nouvelle édition marque un progrès certain sur les précédentes ». 
11. Voir Antonio Casali, Storici italiani fra le due guerre. La "Nuova Rivista Storica» (1917-1943), Napoli, Guida, 1980.

12. AHRF, 9, 1932, p. 221-223.

13. Generale Filareti, Danton e Robespierre (Saggio di psicologia sociale), con introduzione di Corrado Barbagallo, Milano-Roma-Napoli, Società editrice Dante Alighieri, 1922. Voir Luciano Guerci, «Immagini di Robespierre nell'Italia del Novecento», dans Images de Robespierre, Actes du colloque international de Naples, 27-29 septembre 1993, Textes réunis par Jean Ehrard avec le concours d'Antoinette Ehrard et de Florence Devillez, Napoli, Vivarium, 1996, p. 432-435. Cf. aussi Antonio Casali, Storici italiani, op. cit., p. 64 et 67 ; Antonino De Francesco, «Discorsi interrotti. Corrado Barbagallo, Guglielmo Ferrero e la critica della rivoluzione francese » (Nuova rivista storica, 89, 2005, p. 281-340), dans Id., Mito e storiografia della «Grande rivoluzione ». La Rivoluzione francese nella cultura politica italiana del '900, Napoli, Guida, 2006, p. 89-91.

14. AHRF, 9, 1932, p. 223.

15. Ainsi encore Barbagallo : «Mais quelle merveilleuse finesse de recherche et de style dans son effort pour soutenir quelques conclusions presque insoutenables! » (ibid.).

16. Voir Antonino De Francesco, «Discorsi interrotti », op. cit., p. 82-86.

17. Ainsi Walter Maturi en 1952, cité par Antonio Casali, Storici italiani, op. cit., p. 2, note.

18. Ainsi le programme de la Nuova rivista storica, cité ibid., p. 16.

19. Cf. Luciano Guerci, Immagini di Robespierre, op. cit., p. 451 et note 50.

20. C'est l'opinion d'Antonio Casali, Storici italiani, op. cit., p. 130. Sur ses rapports avec Henri Berr, cf. Mario Del Treppo, « La libertà della memoria », dans Marina Cedronio, Furio Diaz, Carla Russo, Storiografia francese di ieri e di oggi, Napoli, Guida, 1977, p. IX-XI.

21. Corrado Barbagallo, Le origini della grande industria contemporanea, 1750-1850. Saggio di storia economico-sociale, Perugia-Venezia, La Nuova Italia, 1929.

22. Il concluait : « Je ne peux pas résumer le livre très riche de M. Barbagallo. Il mérite d'être lu et relu. Sur certains points, on le discutera. Il en vaut la peine ». AHRF, 6, 1929, p. 509-510.

23. Ibid., p. 610-611. Sur sa collaboration à la revue depuis 1927, cf. Delio Cantimori, Storici $e$ storia. Metodo, caratteristiche e significato del lavoro storiografico, Torino, Einaudi, 1971, p. 255-267.

24. Mario Di Gennaro, Giovanni Law e l'opera sua 1671-1727, con introduzione del prof. Corrado Barbagallo, Milano, Società editrice Dante Alighieri, 1931. AHRF, 8, 1931, p. 555 : «sans apporter de révélations, son livre sera utile, parce qu'il est au courant des derniers travaux, notamment de ceux de M. Harsin, et parce qu'il est pourvu d'une bonne bibliographie ».

25. AHRF, 8, 1931, p. 272. L'article de Peroni était publié dans la Nuova rivista storica, janvier-avril 1931.

26. Cf. Antonio Casali, Storici italiani, op. cit., p. 109, 158.

27. Voir par ex. la notice détailléee de la chronique d'un noble de Savone sur les événements de 1798-1806, publiée dans La rivoluzione democratica e l'impero napoleonico a Savona secondo una cronaca contemporanea (Savona, Tip. Savonese, 1929) par Italo Scovazzi et Filippo Noberasco, bibliothécaires et archivistes de Savone : AHRF, 8, 1931, p. 186.

28. Carlo Pellegrini, «Ricordo di Raffaele Ciampini », dans Rassegna storica toscana, XXII, 1976, p. 145-149.

29. Raffaele Ciampini, Napoleone visto dai contemporanei : Thibaudeau, Roederer, Chaptal, Bourrienne, Gourgaud, Torino, F.lli Bocca, 1930.

30. AHRF, 22, 1930, p. 490-491.

31. Antonio Casali, Storici italiani, op. cit., p. 187.

32. AHRF, XV 1938, p. 272-273. Cf. Luciano Guerci, Immagini di Robespierre, op. cit., p. 443 note 39.

33. Raffaele Ciampini, La caduta della monarchia 1792. In appendice lettere inedite degli ambasciatori toscano e piemontese ai loro governi, Bologna, Cappelli, 1934.

34. «Ce sont les dépêches de l'ambassadeur de Toscane à Paris, conservées à Florence et celles de l'ambassadeur de Sardaigne, conservées à Turin. Elles sont reproduites en appendice et 
compteront désormais parmi les sources de l'histoire du 20 juin et du 20 août ». AHRF, 1940, p. 250.

35. $A H R F, 17,1940$, p. 51.

36. Ugo Lenzi, Una lettera inedita di Bonaparte a Marescalchi, 8 brumaire a. 8, Bologna, Tip. Azzoguidi, 1938 : AHRF, 17, 1940, p. 124.

37. Ibid. À la page suivante on signale l'article de Godechot sur le babouvisme et l'unité italienne, Revue des études italiennes, 1939.

38. Pia Onnis Rosa, «Les études italiennes sur l'histoire de la Révolution française de 1940 à 1949 », AHRF, 22, 1950, pp. 358-361.

39. Ibid., p. 360-361.

40. $A H R F, 21,1949$, p. 374-376.

41. AHRF, 21, 1949, p. 281-282.

42. Franco Venturi, Jean Jaurès e altri storici della Rivoluzione francese, Torino, Einaudi, 1948.

43. $A H R F, 21,1949$, p. 373-374.

44. Cf. Anna Maria Rao, "Alessandro Galante Garrone, historien de la Révolution française », art. cit.

45. Alessandro Galante Garrone, « Georges Lefebvre et les historiens italiens », AHRF, 32, 1960, p. 115.

46. Ibid., 22, 1950, p. 78-82. Dans le même numéro on signale l'article de Galante Garrone sur les premiers jacobins piémontais, paru dans Il Ponte, août 1949 (ibid., p. 95).

47. AHRF, 23, 1951, p. 89-92, 93-94.

48. Ibid., p. 92.

49. AHRF, 26, 1954, p. 182-183.

50. Maurice Dommanget, « La Société et les Annales », art. cit., p. 10.

51. Ibid., p. 18, 26.

52. Ibid., p. 22, 25. On ne connaît pas beaucoup de Schifalacqua, dont les AHRF publièrent en 1940 l'article « La révolution française dans les chants de Giosuè Carducci ».

53. Procès verbal de l'Assemblée générale de la Société du 27 avril 1952, présidée par Georges Lefebvre, AHRF, 24, 1952, p. 442-443.

54. Saitta fut sollicité par Lefebvre à commenter, avec Jean Dautry, l'article de Coë «La théorie morellienne et la pratique babouviste", qui fut publié dans les AHRF de 1958, suivi par les observations de Dautry et de Saitta et une réponse de l'auteur. Saitta traduira par la suite ce débat dans ses Ricerche storiografiche su Buonarroti e Babeuf, Roma, Istituto storico italiano per l'età moderna e contemporanea, 1986, p.199-234. Il publia en traduction italienne dans ce même volume, p. 185-197, l'autre article publié dans les AHRF, n 162, 1960, p. 426-435, « Autour de la Conjuration de Babeuf : discussion sur le communisme (1796)».

55. Mais son nom avait été envisagé « au cours des précédentes assemblées » même avant 1958 : AHRF, 30, 1958, p. 96.

56. Walter Markov, Jean-René Suratteau, «Les contributions d'Armando Saitta à l'histoire de la Révolution française vues par un historien allemand et par un historien français », Critica storica, XXVIII, 1991, p. 663.

57. Galante Garrone, "Georges Lefebvre et les historiens italiens », art. cit., AHRF, 32, 1960, p. 115-116.

58. AHRF, 29, 1957, p. 86-87.

59. Le tirage à part est parmi les papiers de Jacques Godechot dans le Centre de documentation historique de Vizille, où on peut consulter de nombreux tirés à part italiens envoyés et parfois dédicacés à Godechot, Soboul, Suratteau.

60. En référence à l'étude de Godechot sur l'unité batave et l'unité italienne.

61. Ibid., p. 88-89.

62. AHRF, 25,1953 , p. 80-82. 
63. Ibid., pp. 82-84.

64. $A H R F, 30,1958$, p. 85-87.

65. AHRF, 32, 1960, p. 335-339.

66. Ibid., p. 339.

67. Ibid., p. 92.

68. «Ce fut justement la réaction morale et politique au fascisme et à ses exaspérations totalitaires et nationalistes, qui fit sentir à quelques-uns de nos historiens le besoin de rechercher les liens ente notre Risorgimento et l'histoire européenne, et, par cette voie, de se rapprocher de la grande Révolution ».

69. Galante Garrone, « Georges Lefebvre », op. cit., p. 109-110.

70. Sur son amitié avec Galante Garrone, cf. Anna Maria Rao, "Alessandro Galante Garrone », art. cit., p. 223.

71. AHRF, 26, 1954, p. 279-280.

72. AHRF, 28, 1956, p. 104-105.

73. $A H R F, 29,1957$, p. 182-185. Sur Carlo Zaghi, je renvoie à Anna Maria Rao, «Il giacobinismo italiano nell'opera di Carlo Zaghi », Studi storici, 45, 2004, p. 47-82.

74. $A H R F, 29,1957$, p. 370-373.

75. AHRF, 36, 1964, p. 507-513.

76. Furio Diaz, v Risposta a Jacques Godechot ", Rivista storica italiana, LXXVII, 1965, p. 702-708.

77. Walter Markov, Jean-René Suratteau, v Les contributions d'Armando Saitta à l'histoire de la Révolution française vues par un historien allemand et par un historien français ", Critica storica, XXVIII, 1991, nº 4, p. 659-666.

78. Ibid., p. 660 .

\section{RÉSUMÉS}

Les rapports entre les AHRF et l'historiographie italienne sont examinés ici en trois étapes. Dans un premier temps, c'est surtout la Nuova rivista storica fondée en 1917 par Corrado Barbagallo qui attire l'attention d'Albert Mathiez, en raison de ses intérêts d'histoire économique et sociale et de sa défense des liens entre Révolution française et Risorgimento italien. La direction de Georges Lefebvre coïncide largement avec l'élan des études italiennes sur la période révolutionnaire dans les milieux de l'exil et de l'anti-fascisme, qui se reflète dans la richesse des notices et des comptes rendus publiés dans la revue. Dans une troisième phase, Jacques Godechot assume un rôle de plus en plus important comme commentateur des ouvrages italiens. Ses commentaires traduisent quelques préjugés et incompréhensions à l'égard de l'historiographie italienne, qu'il considère modelée par Croce. Ils confirment en tout cas la force des liens et des échanges entre les deux historiographies.

The relationship between the AHRF and Italian historiography are examined in this article in three stages. In the first, it was above all the Nuova rivista storica founded 1917 by Corrado Barbagallo that attracted the attention of Albert Mathiez, because of his interest in economic and social history and his defense of the link between the French Revolution and the Italian Risorgimento. In the second, the direction of Georges Lefebvre largely coincided with the burst of Italian studies on the revolutionary period among those in exile and anti-fascist, reflected in the quality of notes and reviews published in the journal. In the third phase, Jacques Godechot 
assumed the increasingly important role as commentator of Italian works. His commentaries contained a few prejudices and misunderstandings about Italian historiography that he considered as shaped by Croce. Still, these phases confirmed the strong relationship and exchanges between the two historiographies.

INDEX

Mots-clés : France, Italie, historiographie, XXe siècle

\section{AUTEUR}

\section{ANNA MARIA RAO}

Dipartimento di Discipline storiche Università di Napoli Federico II Via Marina 3380133 Napoli (Italie) annamrao@unina.it 